\title{
XAS of High Pressure Xe Clusters in Amorphous Carbon and Computational Simulation for the fec and hep Xenon Crystalline Phases
}

\author{
Myriano H. Oliveira Jr and Francisco C. Marques \\ Instituto de Física Gleb Wataghin, Universidade Estadual de Campinas, Caixa Postal 6165, 13083-970 Campinas-SP, Brazil
}

Received on 4 April, 2005

\begin{abstract}
We report the investigation of Xe clusters in amorphous carbon by x-ray absorption spectroscopy (XAS) to understand the properties of solid xenon. Measurements have been performed on xenon $L_{3}$ absorption edge at room temperature (300K). Using computational XANES calculation for fcc and hcp structures it was possible to study the XANES fine structure origin and a relation between the $\mathrm{x}$-ray absorption near edge structure and the lattice constant. Comparing those results with our experimental data we determined that the XAS fine near edge structure has a specific behavior for solid xenon and does not have this behavior for gas Xe end Xe diluted in others chemical elements matrices.
\end{abstract}

Keywords: Xe clusters; Amorphous carbon; X-ray absorption spectroscopy

\section{INTRODUCTION}

Studies on Xe trapped in diamond anvil cells have showed that this element crystallizes in fcc and hcp structures depending on the pressure exerted on it [1,2]. Furthermore, it has been found that solid xenon is an electrical isolating up to $140 \mathrm{GPa}[3-5]$.

There are many works on the study of xenon incorporation in solid crystalline metals or semiconductors by ion implantation [6-10]. It has been observed that when xenon is implanted at room temperature with energies in the range of about 200 to $400 \mathrm{KeV}$ it agglomerates in form of solid crystalline clusters when a concentration of about $4 \%$ xenon is reached. The crystalline cluster phase depends on the host matrix phase, being fcc for fcc and bcc [11-13] matrices and hcp for hcp [14] ones. The xenon lattice constant depends on the chemical nature of the host matrix, once different elements can exerts different pressures on the implanted elements. It has been observed a variation of stress form about few tenths of GPa to $\sim 6 \mathrm{GPa}$ [7-10].

Until the work of R. G. Lacerda et al. [15] about noble gases (NG) solid cluster precipitation in amorphous carbon matrices, nothing was reported on solid NG within amorphous materials using $\mathrm{x}$-ray absorption spectroscopy (XAS) which is one of the most used techniques applied to characterize those clusters in crystalline matrices. From the deposition technique used it is possible to incorporate Xe atoms in amorphous materials during the film growth using an ion gun with energies 100 times smaller than that used for conventional implantation in crystalline materials, obtaining the same Xe concentration (about 5\%) [15].

The appearance of a peak in XANES region after the white line of xenon $L_{3}$ absorption edge, that has been always attributed to xenon in solid phase, remains unexplained. In this work we use some computational calculations on solid crystalline Xe $L_{3}$ XAS spectra and compare them with results from this element implanted in a-C trying to understand the origin of this fine structure and its relation to solid state xenon.

\section{EXPERIMENTAL}

Thin a-C films were deposited by the IBAD (Ion-Beam Assisted Deposition) technique. The apparatus is composed of two Kaufman ion sources, see Fig. 1. One xenon ion beam generated by the gun was used in order to sputter a graphite target with a fixed energy of $1500 \mathrm{eV}$, and a current of $90 \mathrm{~mA}$. The other ion gun (usually called assisting ion gun or assisting ion beam) was used to simultaneously bombard the film during the deposition. The assisting beam energy can be varied from 0 to some hundreds $\mathrm{eV}$ depending on the film structural properties of interest. However, in this work we are interested in studying the sample prepared with a xenon assisting ionic beam with energy of $300 \mathrm{eV}$. This choice is due to the fact that under this condition the films contain xenon clusters in solid phase, as reported elsewhere $[15,16]$. The base pressure in the vacuum chamber was $<10^{-5} \mathrm{~Pa}$, and the total pressure during growth was kept at $6 \times 10^{-2} \mathrm{~Pa}$. The thickness of the film is $70 \mathrm{~nm}$ and was deposited onto silicon wafers at $150^{\circ} \mathrm{C}$. With an assisting ion energy of $300 \mathrm{eV}$ it was obtained a film with intrinsic stress of 10GPa. The intrinsic stress value was obtained using the bending beam method by determining the radius of curvature of the film/substrate, and calculating the stress with the Stoney's equation described in more details elsewhere [17]. The concentration of Xe into the carbon matrix is of about $4 \%$, and was determined by Rutherford Backscattering Spectrometry (RBS).

The X-ray absorption spectroscopy measurements were carried through at SXS (Soft X-ray Spectroscopy) beam line at LNLS (Laboratório Nacional de Luz Síncrotron, Brazil). The measurements were made in the energy range of $4700 \mathrm{eV}$ to $5100 \mathrm{eV}$. The spectra were collected at room temperature $(300 \mathrm{~K})$ and $100 \mathrm{~K}$.

\section{COMPUTATIONAL}

In this work we performed computational calculations of the x-ray absorption spectrum about $L_{3}$ Xe absorption edge in both fcc and hep crystalline phases. The choice of those phases is supported by the fact that they are the two phases 


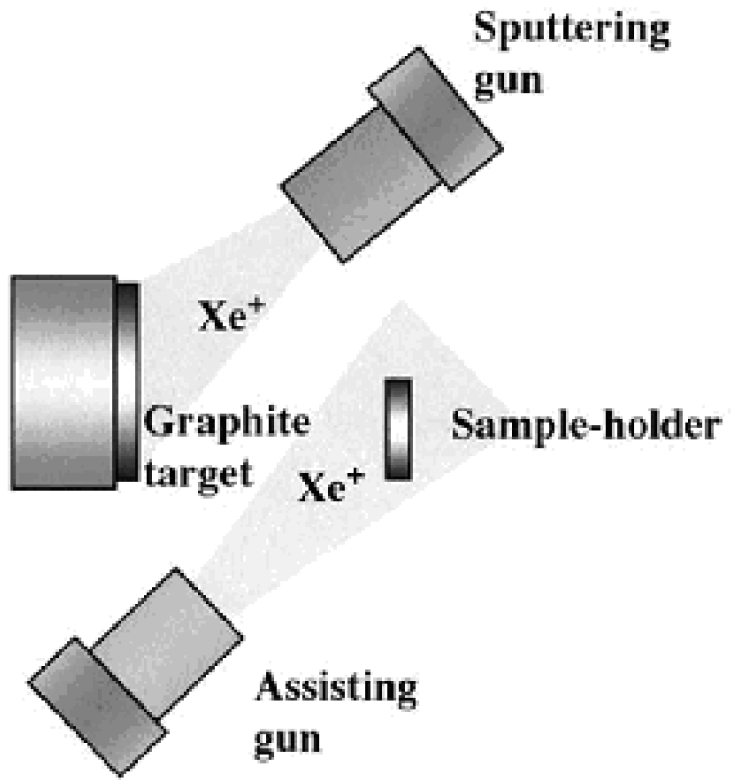

FIG. 1: Ion-Beam assisted deposition system sketch. The assisting beam energy of $300 \mathrm{eV}$ gives a-C films with a xenon concentration of $4 \%$ distributed as solid cluster within the host matrix.

in which this element crystallizes under high pressure, as it has been observed in many works based on x-ray diffraction on xenon trapped in diamond anvil cells [1,2]. Furthermore, there is the observation of fcc and hcp Xe clusters inside crystalline metals and semiconductor matrices when this element is implanted in determined conditions.

Those calculations were done using the FEFF8 code $[18,19]$. The parameters used are basically the defaults ones for FMS, Interstitial and Exchange cards. For SCF card only the convergence factor $c a$ was not the default one (used 0.1). The temperature effects were calculated using the Equation of Motion method in the Debye card. The choice for those conditions is supported by the fact that they reproduce solid krypton and xenon experimental spectra, except for the Fermi level.

\section{RESULTS AND DISCUSSION}

The experimental absorption spectrum obtained for $\mathrm{Xe}$ in amorphous carbon is showed in Fig. 2(a). This spectrum has the same fine structure observed for experimental and simulated (Fig. 2(b)) XANES spectra of solid xenon matrix and solid xenon clusters [10]. This result indicates that the xenon atoms are not randomly distributed inside the carbon matrix but, in the form of clusters in such a way that the interaction $\mathrm{Xe}-\mathrm{Xe}$ within each cluster is stronger then the $\mathrm{Xe}-\mathrm{C}$ interaction. This result is also similar to the results obtained by $\mathrm{G}$. Faraci for Xe implanted in crystalline silicon [10].

The XANES simulation for fcc and hep solid xenon showed the same fine structure, as can be observed in Fig. 3. Even though this fine structure is assumed to be related to solid xenon, since it is not observed in the gas phase or in Xe diluted

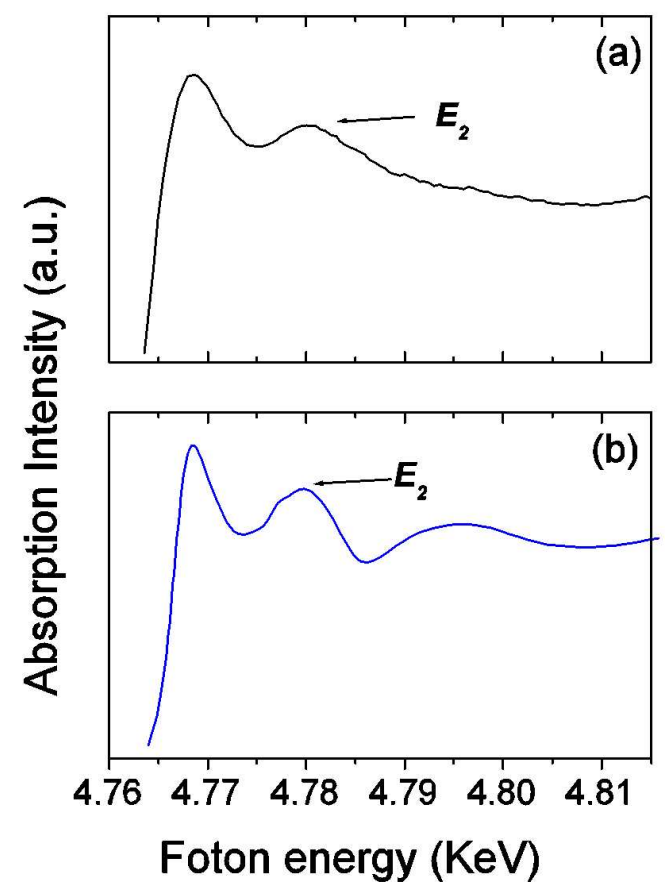

FIG. 2: $\mathrm{L}_{3}$ absorption edge XANES spectra for trapped Xe clusters in a-C matrix (a), and simulation for fcc crystalline $\mathrm{Xe}(\mathrm{b})$. The difference between those spectra in the region of about $4.795 \mathrm{KeV}$ is due to some geometrical factors and the experimental resolution compared to the simulation.

in others matrices, its origin is not known yet. It is known that $\mathrm{Xe} L_{3}$ edge is the electronic transition of a $2 p_{3 / 2}$ electron to the nd levels (and nsalso, but with less contribution) with $\mathrm{n}=5,6 \ldots(=6,7 \ldots$ for $n s)$ [20]. However, as for xenon gas, it was also observed a resonance about $15 \mathrm{eV}$ above the Fermi level, which some authors refers to that fine structure in solid phase to this same effect. This resonance occurs due to double electron monopole excitation in atomic Xe from $5 p$ to $6 p$, i.e., a transition $[2 p, 5 p] \rightarrow[\mathrm{n} d, 6 p][20,21]$. Due to the small cross section compared to the single electron excitation, it is difficult to observe those resonances since its intensity is very small compared to the white line [20]. As a result, the fine structure in XANES region for solid Xe may be not a consequence of multielectron excitation.

When a multi-excitation occurs the electron excited from $2 p_{3 / 2}$ has less energy then those excited in a single electron process. Consequently, this electron stays out of phase with the others, resulting in a reduction of the XAS fine structure amplitude. This effect is considered in FEFF8 calculations in the factor $S_{0}^{2}$ which is called amplitude reduction factor. Thus, the entire XANES region calculation is performed considering single electron excitations with the same reduction factor. Besides the XAS spectra, FEFF8 also allows the calculation of the local projected electronic density of states ( $l$-DOS).

As $\mathrm{Xe} L_{3}$ edge is due the transition $2 p_{3 / 2} \rightarrow$ nd, in Fig. 3 


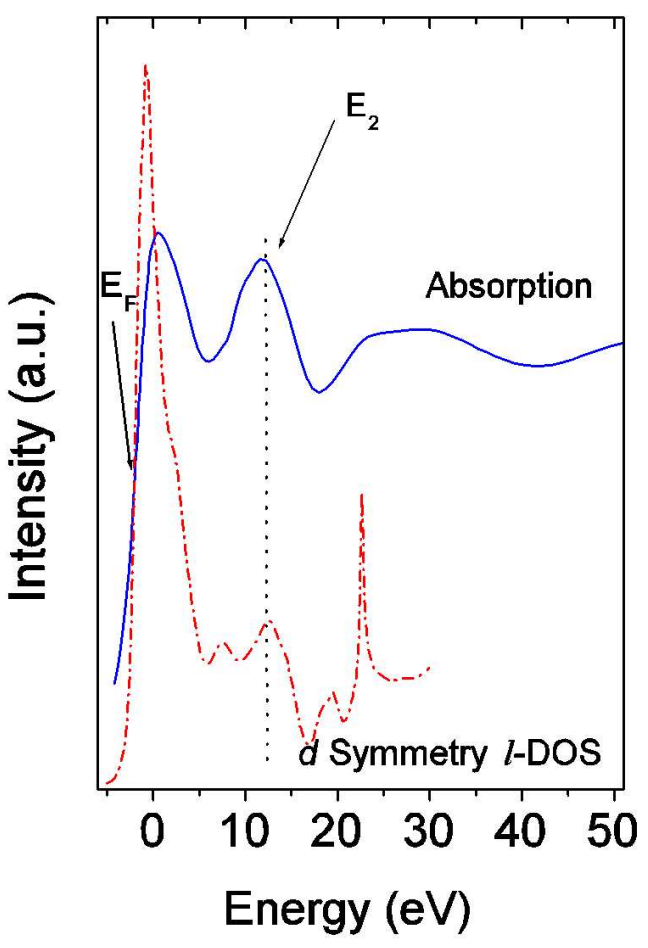

FIG. 3: Simulated XANES spectrum and $d$-symmetry $l$-DOS above Fermi level.

is shown a comparison between the simulated XAS curve and the $l$-DOS with $\mathrm{d}$ symmetry that lies just above the Fermi level $\mathrm{E}_{F}$. In XAS structure we named the maximum energy point of the resonance after the white line, in relation to $E_{F}$, as $E_{2}$. The XAS and $l$-DOS structures have some similarities, such as the white line and $E_{2}$ position.

Figure 4 shows the fine structures of XAS and $l$-DOS structures, as in Fig. 3, in which the atomic contribution was subtracted out and than normalized for graphic purpose. Those curves show the same fine structure which can be explained by their relation [22]:

$$
\begin{aligned}
& \mu(E)=\mu_{0}(E)\left[1+\chi^{\prime}(E)\right] \\
& \rho(E)=\rho_{0}(E)[1+\chi(E)]
\end{aligned}
$$

Where $\mu_{0}\left(\rho_{0}\right)$ is the atomic absorption coefficient (density of states) and the function $\chi$ the fine structure of those signals. The only difference between $\chi$ and $\chi$ ' is that $\chi$ ' is equal to $\chi$ multiplied by the same amplitude reduction factors due to many bodies effects and an excited state time of life.

Thus, the XANES second peak at the $\mathrm{Xe} L_{3}$ edge is due to a single $2 p_{3 / 2}$ electron transition to a $d \quad l$-DOS contribution to the conduction band, i.e. this transition is closely related to the solid xenon band structure or solid xenon clusters. That is why the peak $E_{2}$ does not appear in xenon gas or when one xenon atom interacts only with atoms of another lighter chemical element $[10,15,16]$. However, for elements with atomic

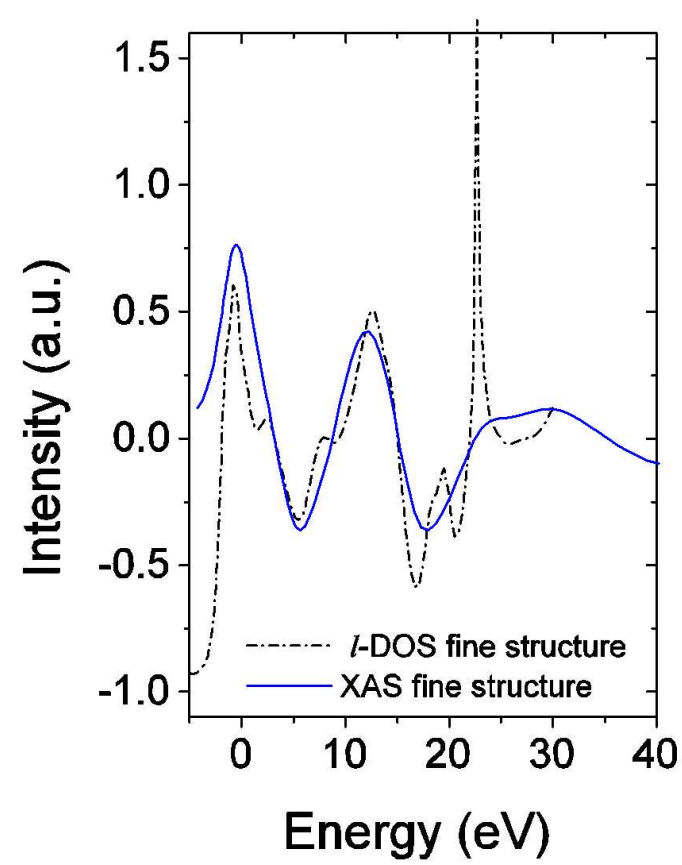

FIG. 4: Simulated XANES spectrum and $d$-symmetry $l$-DOS fine structures.

masses near to that of $\mathrm{Xe}$ as $\mathrm{Ce}$, this peak may appear due to its similar electronic structure, but this hypothesis was not tested yet.

Another interesting result is that the $E_{2}$ position with respect to the Fermi level (EF) is independent of the crystalline phase chosen for the calculations for a fixed first neighbour distances $R$, and for simulations performed for the same $R$ value $\Delta E=E_{2}-E_{F}$ is the same for the experimental data and $\mathrm{fcc}$ and hcp simulation. The noble gas cluster geometry in a-C is still unknown since we are working with an amorphous material and the cluster geometry depends highly on the host matrix structure as it has been showed [11-14]. However, as those films are graphite-like it is reasonable to think that the noble gas clusters are trapped between graphitic planes [15]. Consequently, in this case the clusters would be bi-dimensional and have hexagonal geometry. Thus, $\Delta E$ seems to be independent of the geometry, what would be a possible new method for measuring the interatomic distance for solid xenon without the necessity of knowing the system geometry [23].

\section{CONCLUSION}

With some computational simulations it was possible to explain the origin of the second peak in solid xenon $L_{3}$ absorption edge. Comparing the calculation with experimental results obtained from implanted xenon in amorphous carbon matrix we could verified that the energy of the second peak is independent of the solid xenon geometry. It was also ex- 
plained why this structure does not appear in the Xe gas phase or in Xe diluted, without forming clusters, in solid matrices.

\section{Acknowledgement}

This work was supported by FAPESP, CAPES and CNPq.
We would like to thank the LNLS where the XAS measurements and computational calculations were performed. Thanks are due also to G. Azevedo for fruitful discussions and to LAMFI (Laboratório de Análises de Materiais por Feixes Iônicos - USP) where the RBS measurements were performed.
[1] A. P. Jephcoat, H.-k. Mao, L. W. Finger, D. E. Cox, R. J. Hemley, and C.-s. Zha, Phys. Rev. Lett. 59, 2670 (1987).

[2] H. Cynn, C. S. Yoo, B. Baer, V. Iota-Herbei, A. K. McMahan, M. Nicol, and S. Carlson, Phys. Rev. Lett. 86, 4552 (2001).

[3] R. Reichlin, K. E. Brister, A. K. McMahan, M. Ross, S. Martin, Y. K. Vohra, and A. L. Ruoff, Phys. Rev. Lett. 62, 669 (1989).

[4] K. A. Goettel, J. H. Eggert, I. F. Silvera, and W. C. Moss, Phys. Rev. Lett. 62, 665 (1989).

[5] M. I. Eremets, E. A. Gregoryanz, V. V. Struzhkin, H.-k. Mao, R. J. Hemley, N. Mulders, and N. M. Zimmerman, Phys. Rev. Lett. 85, 2797 (2000).

[6] D. I. Potter and C. J. Rossouw, J. Nucl. Mater. Science 161, 124-131 (1989).

[7] A. Vom Felde, J. Fink, Th. Müller-Heinzerling, J. Pflüger, B. Scheerer, G. Linker, and D. Kaletta, Phys. Rev. Lett. 53, 922 (1984).

[8] C. Templer, H. Garem, J. P. Riviere, and J. Delafond, Nucl. Instrum. Meth. In Phys. Research B 18, 24-33 (1986).

[9] C. Templer, H. Garem, and J. P. Riviere, Philos. Mag. A 53 , 667-675 (1986).

[10] G. Faraci, A. R. Pennisi, and A. Terrasi, Phys. Rev. B 38, 13468 (1988).

[11] H. H. Andersen, J. Bohr, A. Johansen, E. Johnson, L. Sarholt-
Kristensen, and V. Surganov, Phys. Rev Lett. 59, 1589 (1987).

[12] J. H. Evans and D. J. Mazey, J. Phys. F 15, L1 (1985).

[13] R .C. Birtcher and Jäger, Nucl. Instrum. Methods B 15, 435 (1986).

[14] J. H. Evans and D. J. Mazey, J. Nucl. Mater. 138, 176 (1986).

[15] R. G. Lacerda, M. C. dos Santos, L. R. Tessler, P. Hammer, F. Alvarez, and F. C. Marques, Phys. Rev. B 68, 054104 (2003).

[16] M. H. Oliveira Jr., P. F. Barbiere, R. G. Lacerda, and F. C. Marques, Thin Sol. Films 469-470, 112 (2004).

[17] M. M. de Lima, Jr., R. G. Lacerda, J. Vilcarromero, and F. C. Marques, J. Appl. Phys. 86, 4936 (1999).

[18] A. L. Ankudinov, B. Ravel, J. J. Rehr, and S. D. Conradson, Phys. Rev. B 58,7565 (1998).

[19] A. L. Ankudinov, and J. J. Rehr, Phys. Rev. B 62, 2437 (2000).

[20] I. Arcon, A. Kodre, M. Stuhec, and D. Glavic-Cindro, Phys. Rev. A 51, 147 (1995).

[21] K. Zhang, E. A. Stern, J. J. Rehr, and F. Ellis, Phys. Rev. B 44 2030 (1991).

[22] A. L. Ankudinov, B. Ravel, J. J. Rehr, and S. D. Conradson, Phys. Rev. B 58, 7565 (1998).

[23] M. H. Oliveira Jr and F. C. Marques, to be published. 